\title{
La importancia de las emociones para la neurodidáctica
}

\author{
The importance of emotions for the neurodidactics
}

\author{
Verónica Benavidez, Magister ${ }^{l}$ \\ Ramón Flores, Magister ${ }^{2}$
}

\begin{abstract}
RESUMEN
Este artículo pretende exponer la importancia de las emociones para la neurodidáctica en la medida que éstas inciden en el proceso de aprendizaje. El objetivo de la neurodidáctica es potenciar el desarrollo de nuevas estrategias basadas en las teorías de las neurociencias, que dan cuenta del manejo de las emociones y su relación con el aprendizaje, mismo que se puede conceptualizar como la creación de memorias impulsadas por estímulos que lo potencian o inhiben. El aprendizaje tiene relación directa no solo con la memoria, sino fundamentalmente con los estímulos emocionales, de los que depende qué y con qué profundidad se aprende. El aprendizaje inicia en el hipocampo y amígdala, que colaboran en la evaluación de los estímulos emocionales. Las estrategias didácticas que se utilizan en el aula tienen la capacidad de favorecer el aprendizaje de los estudiantes o de impedirlo, en función de la gestión de las emociones que el maestro realice. El artículo se construyó sobre la base de la revisión de literatura relacionada con el funcionamiento del cerebro emocional, y la forma en que las emociones influyen en el aprendizaje humano.
\end{abstract}

Palabras clave: Neurociencias, neuroeducación, neurodidáctica, emociones.

\begin{abstract}
This article exposes the importance of emotions for the neurodidactics as they affect the learning process. The objective of the neurodidactics is to promote the development of new strategies based on the theories of neurosciences, that shows the manage of emotions and their relationship with the learning process, which can be conceptualized as the creation of memories driven by stimuli that boost or inhibit. Learning has a direct relationship not only with memory, but fundamentally with emotional stimuli, on which depends what and how deep is the student learning. Learning begins in the hippocampus and amygdala, which collaborate in the evaluation of emotional stimuli. The didactic strategies used in the classroom contribute to the learning process of students or to stop it, depending on the management of the emotions that the teacher make. The article was built on the basis of the review of literature related to the functioning of the emotional brain, and the way in which emotions influence human learning.
\end{abstract}

Key Words: Neuroscience, neuroeducation, neurodidactics, emotions.

\footnotetext{
${ }^{1}$ Pontificia Universidad Católica del Ecuador. Docente Facultad de Psicología. Ecuador. Correo electrónico: veronicavboa@yahoo.com

${ }^{2}$ Universidad Central del Ecuador. Docente Facultad de Filosofía, Letras y Ciencias de la Educación. Ecuador. Correo electrónico: ramonfloresp@yahoo.es Recepción: 8/10/2018 Aceptación: 3/1/2019
} 


\section{Introducción}

Los resultados de las investigaciones actuales en el campo de las Neurociencias han demostrado la importancia que las emociones tienen en procesos como el aprendizaje, la toma de decisiones y la percepción, entre otros (Blanco, 2014); sin embargo, en los procesos de enseñanza y aprendizaje aún no se incluyen de manera generalizada, estrategias didácticas basadas en el manejo de las emociones, que permitan potenciar la adquisición de nuevos conocimientos. Tomando en cuenta que algunas investigaciones han demostrado que tanto el cerebro en estado de maduración, como uno maduro, modifican su estructura a partir de nuevos aprendizajes según Bransford, Brown y Cocking (citado en Mogollón, 2010), y que según Coleman (citado en Mogollón, 2010) el aprendizaje no está separado de las emociones, por lo que trabajar en el desarrollo de la inteligencia emocional, con el objetivo de que los estudiantes comprendan y traten con sus emociones, es fundamental para un aprendizaje duradero.

Según Blanco (2014, pp.182-200) se entiende por aprendizaje al "proceso de adquisición de nueva información, y por memoria a la persistencia del aprendizaje en un estado que puede revelarse en cualquier momento dado". Estos dos procesos están relacionados con las emociones ya que dependen de su intensidad, por lo que es necesario proponer métodos o estrategias para lograr la intensidad adecuada de las emociones, de manera que incidan positivamente en el mejoramiento de los aprendizajes.

Las estrategias didácticas relacionadas con el manejo de las emociones deben ser propuestas de manera sencilla, cercana a los educadores, con ejemplos prácticos y novedosos que puedan ser aplicados en el aula. Es importante que se tome en cuenta a la hora de enseñar (Totger, 2017, p. 19) que la motivación, repetición no mecanizada sino reflexiva, así como la utilización de variados estímulos multisensoriales y entornos resonantes son fundamentales para la fijación de los nuevos aprendizajes.

Los estados emocionales de los estudiantes son básicos para el aprendizaje, lo que significa que los docentes deben estar conscientes plenamente de que deben saber leer estas emociones, y además provocar aquellas que resultan positivas para la adquisición y fijación de los conocimientos. Por otro lado, los estudiantes deben aprender a gestionar 
sus emociones, lo que implica que se potencie el desarrollo de la inteligencia emocional de manera consciente.

El estudio consistió en una investigación documental - descriptiva, elaborada sobre la base de la revisión de literatura, organizada, sistematizada y analizada en función de la temática; los documentos revisados corresponden a un conjunto de artículos científicos, tesis de doctorado, libros y textos, impresos o digitales, entrevistas publicadas online y ponencias, que fueron analizadas, y de las que se recuperó la información pertinente relacionada con las ciencias cognitivas, neuroeducación y neurodidáctica.

Las fuentes que sirvieron de base para el estudio se localizaron en Digitalia Hispánica, EBSCO Ebooks, E-libro y diferentes buscadores, bajo las siguientes palabras clave: neurociencias, cerebro triuno, inteligencia emocional, cerebro emocional, sistema límbico, amígdala, emoción, percepción, atención, motivación, memoria, procesos cognitivos, didácticas emocionales, aprendizaje humano, neurodidáctica y neuroeducación. La investigación cubrió el período 1995-2018.

Queda clara la necesidad de proponer una diversidad de estrategias de enseñanza y aprendizaje que propicien la adquisición duradera de nuevos conocimientos, basadas en el manejo de las emociones, desde el reconocimiento por parte de los maestros, como la práctica por parte de los estudiantes.

\section{Desarrollo}

\subsection{El cerebro triuno de Paul Mac Lean}

\section{El Cerebro Triuno}

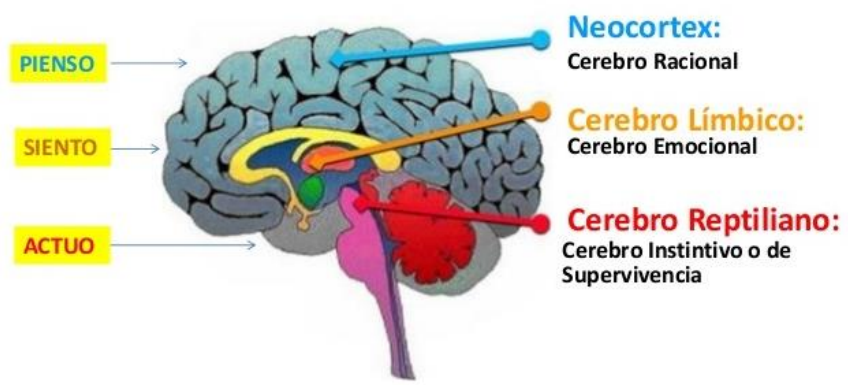

Fuente: https://blog.aspiria.mx/wp-content/uploads/2016/02/coaching-10-638-1.jpg Recuperado 28-12-18 
La teoría del cerebro triuno propuesta por Maclean y posteriormente retomada por Beauport se centra en el ser humano como un ser con múltiples capacidades interconectadas y complementarias; según esta teoría se puede analizar el comportamiento humano de manera integral, donde la cognición, la emoción y la conducta humana trabajan al unísono e influyen definitivamente en el desempeño del sujeto en cualquier ámbito.

Mac Lean (s.f., citado por LeDoux, 1999) introdujo la teoría del cerebro ternario compuesto de tres capas, la más antigua llamada cerebro reptil, cubierta por la segunda capa, el cerebro mamífero, también llamado emocional y éste a su vez por el cerebro de formación más reciente, el cerebro humano. "Cada tipo de cerebro, según MacLean, tiene su propia inteligencia, su propia memoria particular, y su propio sentido del tiempo y del espacio, así como sus propias funciones motoras y de otra clase” (Ledoux, 1999, p. 108).

El cerebro emocional se encuentra formado por el sistema límbico (ubicado detrás de la nariz y abarca hasta los occipitales) tiene que ver con la respiración, la rabia, el amor, los estados de ánimo, el afecto (Freilich, 1989). El sistema límbico es el paso obligado al neocortex en donde se procesa la información, una vez que ha sido filtrada por el cerebro emocional.

El cerebro mamífero o paleomamífero llamado también cerebro emocional está compuesto por el sistema límbico, que no es una región exactamente delimitada del cerebro, sin embargo en la mayoría de literatura correspondiente se menciona que está conformado por el hipocampo, la amígdala cerebral, el hipotálamo, el fornix y los cuerpos mamilares; "La amígdala (...) está implicada en las respuestas emocionales: los sentimientos, la expresión de la emoción, los recuerdos de las emociones y el reconocimiento de los signos de la emoción de los demás" (Aguilar, 2011).

Del sistema límbico la amígdala es muy importante porque es la responsable de asociar cargas emocionales a los recuerdos de largo plazo, para evitar experiencias dolorosas, o por el contrario reincidir en algunos comportamientos que han provocado placer o alegría.

\subsection{Neurociencias, neuroeducación y aprendizaje}

Las neurociencias se refieren al estudio del sistema nervioso, desde diversos enfoques y a través de diferentes disciplinas, desde la Biología Molecular, la Fisiología, 
la Genética, la Psicología, entre otras (Velásquez, et al., 2009). Para Kandel (citado en Salas, 2003) las neurociencias "son un conjunto de ciencias que estudian el sistema nervioso, principalmente cómo la actividad del cerebro se relaciona con la conducta y el aprendizaje".

"La neurociencia es la disciplina encargada de estudiar el cerebro y como éste da origen a la conducta y el aprendizaje" (Marueira, 2010, p.1); las neurociencias se ocupan además de estudiar la plasticidad del sistema nervioso, de la importancia del ambiente en el aula, las bases de la motivación, la atención, las emociones y la memoria, como constituyentes esenciales del proceso de enseñanza-aprendizaje". En este caso Marueira se refiere a la neuroeducación y a la neurodidáctica como disciplinas que se encargan de la relación enseñanza-aprendizaje y cerebro.

La relación entre aprendizaje, enseñanza y neurociencia se conoce como neuroeducación, misma que según De la Barrera (2009), se encarga del desarrollo de la neuromente durante la escolarización. La neuroeducación es una ciencia cuyo objeto de estudio es una nueva visión de la enseñanza basada en el cerebro, en los conocimientos que sobre su estructura y funcionamiento se tienen al momento.

El objetivo de la neuroeducación es fomentar el desarrollo de la memoria por parte de los estudiantes, así como proponer estrategias didácticas que ayuden a los docentes en el proceso de enseñanza, estrategias que tomen en cuenta la importancia de las emociones como mediadoras del aprendizaje, en función de los impulsos positivos o negativos que fortalezcan o inhiban el mismo. Es básico en el contexto de la neuroeducación que los docentes tengan pleno conocimiento de cómo funciona el cerebro, la memoria, la atención y las emociones, cómo éstas se desarrollan y cómo participan en el aprendizaje. "Todo educador debe saber cómo es el cerebro, cómo aprende, cómo procesa, registra, conserva y evoca una información, entre otros aspectos" (Campos, 2014, p.15).

"El propósito de la neuroeducación es elucidar las estructuras y funciones cerebrales asociadas con la educación" (Gabrieli, citada en Bruer, 2016, p.8), "la NE puede decirnos en qué dirección buscar intuiciones sobre el modo de mejorar la enseñanza y el aprendizaje; pero no le dice al educador o al diseñador de políticas cómo aplicar esas intuiciones en los contextos educativos" (Howard Jones et al., citado en Bruer, 2016, p.8), y "la psicología cognitiva ofrece una base de conocimientos con la cual mejorar los resultados educativos" (Bruer, 2016). Entonces es imperativa una investigación acerca de los métodos didácticos (neurodidáctica) que permitan la aplicación práctica de las 
intuiciones, y conocimientos propuestos tanto por la NE, como por las neurociencias en general y de la psicología cognitiva en particular.

El aprendizaje es un producto del cerebro, a la vez que éste mejora con el aprendizaje, por lo que se dice que lo que mejor hace el cerebro es aprender; su papel primordial es la creación de redes neuronales que se modifican continuamente en función del entorno, lo que más estimula al cerebro es la novedad, los cambios, lo desconocido. Para Jensen (2010), el cerebro se utiliza cada vez menos cuando se ejercita, el estímulo refuerza el aprendizaje, por esa razón los novatos utilizan más el cerebro.

\footnotetext{
El cerebro humano es un órgano biológico y social que se encarga de todas las funciones y procesos relacionados con el pensamiento, la intuición, la imaginación, la lúdica, la acción, la escritura, la emoción, la conciencia e infinidad de procesos que, gracias a la plasticidad entendida como la capacidad que posee el cerebro para cambiar respondiendo a las modificaciones del entorno, puede modificar las conexiones entre neuronas, la red de capilares que les proporcionan oxígeno y nutrientes y producir nuevas neuronas, todo ello, durante la vida de la persona y no solamente en la adolescencia o los primeros años de adultez como se creía anteriormente (Velásquez et al. 2009, p. 334).

El cerebro es la máquina gracias a la cual se producen todas las formas de aprendizaje (...) el cerebro es también el mecanismo natural que pone límites en el aprendizaje. Determina lo que puede ser aprendido, cuánto y con qué rapidez (Blakmore y Frith, 2010, p.19).
}

La plasticidad del cerebro es un concepto muy importante para la educación, ya que el aprendizaje lo modifica, y a la vez el cerebro modificado aprende con más facilidad nuevos conocimientos. Morgado (2005, p.221) afirma que "Cuanta más plasticidad tiene su sistema nervioso más posibilidades de aprendizaje tiene un animal. Por tanto, el aprendizaje puede considerarse como un cambio en el sistema nervioso".

El cerebro tiene una enorme capacidad de reorganizarse y adaptarse al entorno ya que su enorme plasticidad así lo permite, esta realidad debe ser aprovechada por los docentes a través de estrategias que utilicen la novedad y curiosidad que tanto estimula la aparición y reorganización de redes neuronales (Blakemore y Frith, 2010). A decir de Codina (2015, p.19) "la plasticidad del cerebro significa que su estructura y conectividad neuronal cambian con la experiencia".

El modelo de enseñanza que se utiliza actualmente debe ser cambiado por otro en el que se tenga en cuenta cómo aprende el cerebro, en el que los docentes conozcan cómo se producen de manera más efectiva los aprendizajes explícitos basados en la atención 
selectiva sostenida y consciente; que son resultado de clases participativas, en donde la práctica es más habitual, y el ejercicio de enseñanza mutua entre pares es el que prima (Labus y Galbán, s.f.).

El cerebro aprende mejor cuando el clima de la clase es relajado pero desafiante, las amenazas, desinterés, falta de atención inhiben el aprendizaje. Cada cerebro es diferente, su biología depende del contexto de crianza, sea este enriquecido o empobrecido, su desarrollo definirá los gustos o aversiones de cada persona, y por tanto el aprendizaje debe responder a las necesidades emocionales de cada aprendiz.

\subsection{Las emociones como motor de aprendizaje}

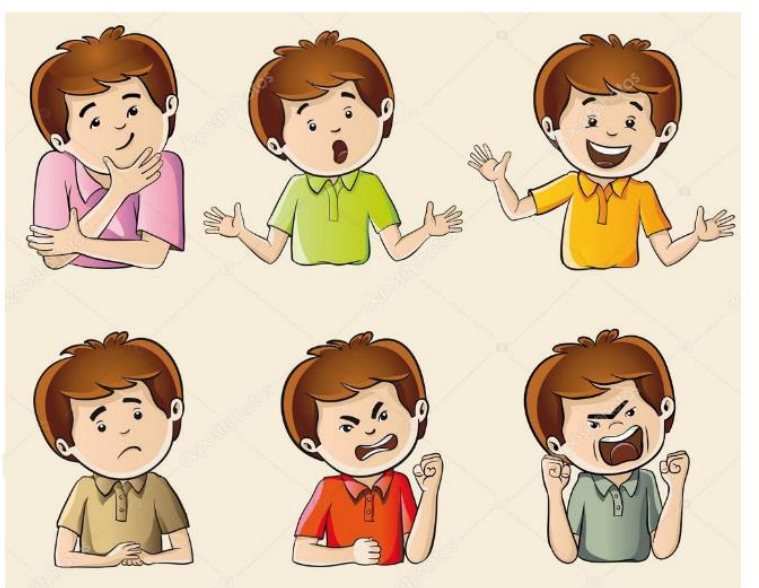

Fuente: https://st2.dep
Recuperado: $28-12-18$

"Una emoción es un grupo de tres clases de respuestas distintas pero interrelacionadas: respuestas fisiológicas, conductas manifiestas y sentimientos conscientes" (Gluck, et al., 2009, p.409). Las emociones "son reacciones psicofisiológicas que representan modos de adaptación a ciertos estímulos del individuo cuando percibe un objeto, persona, lugar, suceso o recuerdo importante (...) y nos preparan para una reacción” (Rotger, 2017, pp. 23). Las emociones son fundamentales para la supervivencia y se controlan en el sistema límbico, también denominado cerebro medio, emocional o mamífero, de acuerdo a la teoría del cerebro triuno propuesta por Paul MacLean (s.f., citado en LeDoux, 1999); ellas pueden ser controladas ya que el sistema límbico y el neocortex trabajan conjuntamente (Goleman, 1996).

El sistema límbico es el responsable del desarrollo de las emociones y motivaciones, en su estructura se encuentran: la amígdala y el hipocampo, relacionados 
con la memoria, quienes determinan qué recuerdos y en qué parte del hemisferio cerebral se almacenan a largo plazo.

La amígdala además registra y analiza los estímulos recibidos a través del tálamo desde el exterior, si son negativos inhiben el paso de información a los lóbulos prefrontales, de esta manera el aprendizaje no se lleva a cabo. La amígdala es uno de los órganos más importantes para el aprendizaje, ya que decide el carácter de las reacciones (positivas o negativas) ante la información que ingresa al cerebro a través de cualquier estímulo, "Este órgano es crucial en el contexto de aprendizaje porque bloquea varios aspectos de aprendizaje si hay emociones amenazantes" (De la Cuesta, 2016, p.10).

Investigaciones hechas en laboratorios demuestran que las zonas más ligadas a la humanidad y al comportamiento civilizado tienen relación directa con el lóbulo frontal, encargado del juicio, el control de impulsos y la planificación, entre otras funciones, y estas zonas aparecen activadas mayormente a medida que crecemos; por el contrario, en el niño y en el adolescente hay un predominio de zonas límbicas (LeDoux; citado en Arboccó de los Heros, 2015, p.2).

Las dos estructuras del sistema límbico: la amígdala y el hipocampo están relacionadas directamente con la corteza neofrontal, si por alguna razón resultan inhibidas a causa del miedo o una situación de estrés puede existir una disminución en el aprendizaje (Mogollón, 2010). El sistema límbico se complementa con la neocorteza, la amígdala y los lóbulos prefrontales, su actuación coordinada y positiva permite que la inteligencia emocional mejore y como resultado mejora también la inteligencia cognitiva (Goleman, 1996).

Las emociones están presentes en todos nuestros aprendizajes, implícitos o explícitos, los potencian o inhiben. "Las emociones son reacciones psicofisiológicas que representan modos de adaptación a ciertos estímulos del individuo cuando percibe un objeto, persona, lugar, suceso o recuerdo importante” (Rotger, 2017, p.23). Las emociones son inevitables y se manifiestan a través de las sensaciones, que son reacciones fisiológicas; cuando se interpretan estas sensaciones y se les puede poner un nombre se dice que se produce un sentimiento, mismo que es opcional (Rotger, 2017).

El aprendizaje emocional incluye el condicionamiento clásico y el condicionamiento instrumental, es decir que las respuestas emocionales pueden ser aprendidas, y en este proceso es fundamental el papel de la amígdala (Guck, 2009). 
Cuando los sentimientos son ignorados pueden actuar inadvertidamente y en consecuencia desconocer influencias positivas o negativas. Cuando hay un desborde de nuestro cerebro emocional, nuestro cerebro de trabajo puede tener poca capacidad de atención para tener en mente los hechos necesarios para terminar una tarea, la adquisición de un concepto o la toma de una decisión inteligente (De la Barrera y Donolo, 2009, p.11).

"Las informaciones, a las que el sistema límbico ha impreso su sello emocional, se graban profunda y perdurablemente en la memoria" (Gerhard y Gerhard, 2003, p. 43). Las emociones fomentan el aprendizaje cuando pueden estimular toda actividad a nivel de redes neuronales intensificando las conexiones sinápticas, por lo que es mejor el aprendizaje cuando están involucradas las emociones (Immordino-Yang y Damasio, 2007; citado en Mogollón, 2010).

En la actualidad se está investigando el efecto de la violencia verbal y psicológica, que a decir de Baroccó de los Heros (2015), está retrasando el desarrollo normal del cerebro de los infantes, y provocando en los niños dificultades para el autocontrol, el aprendizaje y el equilibrio emocional. Así al parecer los métodos de enseñanza basados en el castigo o violencia de cualquier tipo, no solo afectan emocionalmente a los estudiantes, sino que retrasan su desarrollo normal; lo que podría significar, que si bien el aprendizaje es mejor cuando están involucradas las emociones, será peor si éstas son negativas. La atención puede ser fija, como la que se genera ante un posible peligro; puede ser orientativa, como reconocer una cara entre mil; o puede ser ejecutiva, que es lo que requiere el estudio, ya que hay necesidad de pasar de un concepto a otro en un período de tiempo corto, además está la atención difuminada, que es la que tienen los genios (...) (Mora, 2013).

La atención puede verse empañada por distintas emociones como la rabia, el miedo, el enfado, la tristeza, frustración, entre otras, y en esas circunstancias es difícil aprender.

La motivación y emoción dirigen el sistema de atención, el cual decide qué informaciones se archivan en los circuitos neuronales, y por tanto, qué se aprende (Posner \&Posner, y Rothbart; citados por De la Barrera y Donolo, 2009). 


\subsection{La atención, función indispensable para el aprendizaje}

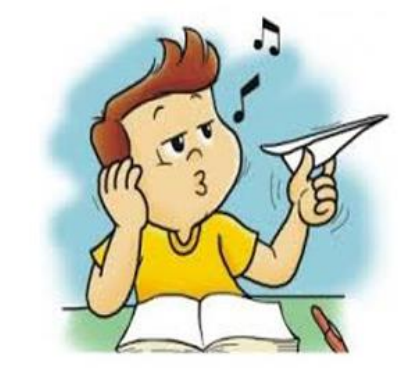

Fuente: http://mcarmenalonso.es/wp-content/ uploads/2015/08/taller-atencion.jpg Recuperado: $20-12-2018$

"La atención puede ser definida como la capacidad para concentrarse en un estímulo que llega al cerebro mientras ignora otros estímulos" (Codina, 2014, p. 65). La atención es un proceso cognitivo muy importante, ya que tiene la capacidad de permitir o impedir la participación de estímulos sensoriales internos o externos en el proceso de aprendizaje. La atención requiere de ciertos neurotransmisores producidos por aminoácidos que le suministran las proteínas, lo que sugiere que una alimentación rica en proteínas puede facilitar el mantenimiento de la atención; los neurotransmisores responsables de la atención están presentes más en períodos matutinos, por lo que esta realidad debe ser tomada en cuenta por los docentes para la organización del currículo (Spenger, citado en Salazar, 2005).

La atención es de suma importancia para el proceso de aprendizaje, especialmente la denominada atención ejecutiva, cuyo pico tope es de 20 minutos, tiempo que el docente debe aprovechar al máximo en el proceso de enseñanza.

Para que la atención se active es necesario que se produzca algún hecho novedoso, interesante o curioso. "Nada se puede aprender ni memorizar a menos que exista el calor emocional que lo sostenga" (Souza, 2017, p.13), y la emoción aparece cuando la monotonía desaparece. El cerebro es un apasionado de las novedades, está en constante busca de estímulos, cuando no los encuentra se sumerge en sí mismo para buscar nuevas sensaciones (Souza, 2014). "La novedad supone aquello que no encaja en los patrones establecidos, de modo que reclama la atención del que aprende" (Codina, 2014, p.64).

La atención puede ser fija, como la que se genera ante un posible peligro; puede ser orientativa, como reconocer una cara entre mil; o puede ser ejecutiva, que es lo que requiere el estudio, porque hay que pasar de un concepto a otro en un período de tiempo 
corto, además está la atención difuminada, que es la que tienen los genios (...) (Mora, 2013). Los docentes por tanto deben conocer y sacar provecho a los estímulos que provocan una atención ejecutiva, proponer acciones novedosas que inquieten a los estudiantes y favorezcan los debates que incluyan temas de su interés, y relacionarlos con los temas de tratamiento obligatorio.

La atención puede verse empañada por distintas emociones como la rabia, el miedo, el enfado, la tristeza, frustración, entre otras, y en esas circunstancias es difícil aprender.

La motivación y emoción dirigen el sistema de atención, el cual decide qué informaciones se archivan en los circuitos neuronales, y por tanto, qué se aprende (Posner \&Posner, y Rothbart; citados por De la Barrera y Donolo, 2009).

A menudo los estudiantes llegan a las aulas cargados de emociones negativas, resultantes de problemas familiares o decepciones personales, en esos casos es difícil llamar su atención, peor aún mantenerla, es entonces cuando el maestro debe ensayar acciones novedosas que motiven a los aprendices y les hagan olvidar su frustración, de manera que se puedan producir aprendizajes duraderos.

\subsection{La memoria, otra cara del aprendizaje}

Para (Morgado, 2005) no hay memoria sin aprendizaje, ni aprendizaje sin memoria, lo que hace de la memoria una de las herramientas básicas para el aprendizaje, sin la cual aprender sería una tarea imposible. La memoria permite almacenar información y recuperarla cuando es preciso, permite revivir el pasado y evocar las sensaciones y emociones que experimentamos, y lo más importante, permite realizar acciones que aprendimos en el pasado y activar rutinas apropiadas al contexto (Bajo et. al, 2016). "El cerebro utiliza distintos sistemas de memoria para recibir y procesar la información de muy diversos modos, como, por ejemplo, la memoria emocional, la espacial, la de trabajo, etc.” (Codina, 2014, p.66).

En el proceso de aprendizaje la memoria juega un papel muy importante, ya que permite aprender nueva información y almacenarla para recuperarla cuando sea necesario. La memoria es de tres tipos: sensorial, de corto plazo (operativa o de trabajo) y largo plazo; la memoria sensorial tiene una duración de entre 200 y 300 milisegundos, 
tiempo después del que la información, visual, olfativa, auditiva, etc. desaparece o se transmite a la memoria de corto plazo para su procesamiento.

La memoria de corto plazo es fundamental para el procesamiento cognitivo, ya que realiza dos funciones, mantiene la información presente en la mente y permite su manipulación en otros procesos cognitivos superiores como el razonamiento, comprensión y resolución de problemas, este tipo de memoria es similar a la memoria RAM de un computador, mantiene presente la información mientras se realizan las diferentes operaciones, y se borra si no se grava en el disco duro, en nuestro caso en la memoria de largo plazo.

"La memoria de trabajo se puede considerar un sistema generalizado de control cognitivo y ejecutivo que guía el comportamiento y que implica interacciones entre los diversos procesos mentales (atención, percepción, motivación, emociones y memoria)" (Morgado, 2005, p. 8), este tipo de memoria es una suerte de coordinador de los procesos que intervienen en la realización de diferentes acciones como el aprendizaje.

La memoria de largo plazo almacena la información previamente seleccionada, de manera duradera y se lleva a cabo cuando los aprendizajes son significativos (Codina, 2014), por lo que es fundamental aplicar estrategias didácticas en el aula que permitan a los estudiantes apropiarse de los aprendizajes, y esto se logrará únicamente cuando estén implicadas positivamente las emociones, es decir que los materiales de estudio sean interesantes y motivadores. La memoria de largo plazo es de dos tipos: implícita o procedimental y explicita o declarativa.

La memoria implícita o procedimental corresponde a los recuerdos inconscientes en los que se basan los hábitos perceptivos y motores; almacena la información de manera inconsciente, es automática y está relacionada con el aprendizaje de habilidades rutinarias; así por ejemplo montar bicicleta, manejar un automóvil o vestirse; la memoria explícita es la suma de recuerdos de las experiencias y conocimientos adquiridos a propósito (Morgado, 2005), como realizar las operaciones matemáticas básicas, para lo cual recordamos conocimientos adquiridos en los primeros años de escuela, o escribir un ensayo sobre la base de un esquema previamente comprendido y memorizado.

La memoria explicita o declarativa está asociada a la percepción consciente, es de dos tipos: memoria semántica y memoria episódica.

Sprenger, (1991; citado en Salazar, 2005, p. 9) señala que “(...) la memoria episódica se asocia con el recuerdo del contexto, lo espacial, la ubicación y la memoria 
automática (...) y que está referida a la memoria de respuesta condicionada", además se relaciona con el recuerdo de hechos concretos como por ejemplo con el nacimiento de un hijo, la graduación de la universidad o el primer día de trabajo.

La memoria semántica está relacionada con el uso del lenguaje, este tipo de memoria almacena el conocimiento del lenguaje y el mundo, sin tener en cuenta cómo se aprendió, también los materiales visuales, figuras o gráficos. Los procesos de memoria y aprendizaje están relacionados directamente con las redes neuronales que el cerebro produce, a más aprendizaje más redes neuronales se construyen, mismas que a la vez potencian nuevos aprendizajes.

Francisco Mora en el prólogo del libro "Implicar al cerebro reconectado" manifiesta que aprender y memorizar consiste en "cambiar el cableado del cerebro" (Sousa, 2018), las conexiones neuronales se fortalecen o desaparecen según se utilicen; por lo que memorizar permite reforzar dichas conexiones hasta que se vuelven automáticas, y esto será posible cuando se utilicen estrategias de aprendizaje que permitan reiterar los nuevos conocimientos paulatinamente hasta que se fijen en la memoria.

Finalmente, y quizá lo más importante, la memoria está ligada a la emoción, ya que ésta última no solo influye en la forma como se almacena sino también como se recuerda (Guck, 2009), las memorias más intensas están asociadas con emociones fuertes, de donde se deduce que aprender debe ser el resultado de emocionarse de manera continua y positiva, y que por tanto el docente debe suscitar en sus clases emociones placenteras, para que sus enseñanzas sean recordadas de manera más prolongada.

\subsection{El aprendizaje y las emociones}

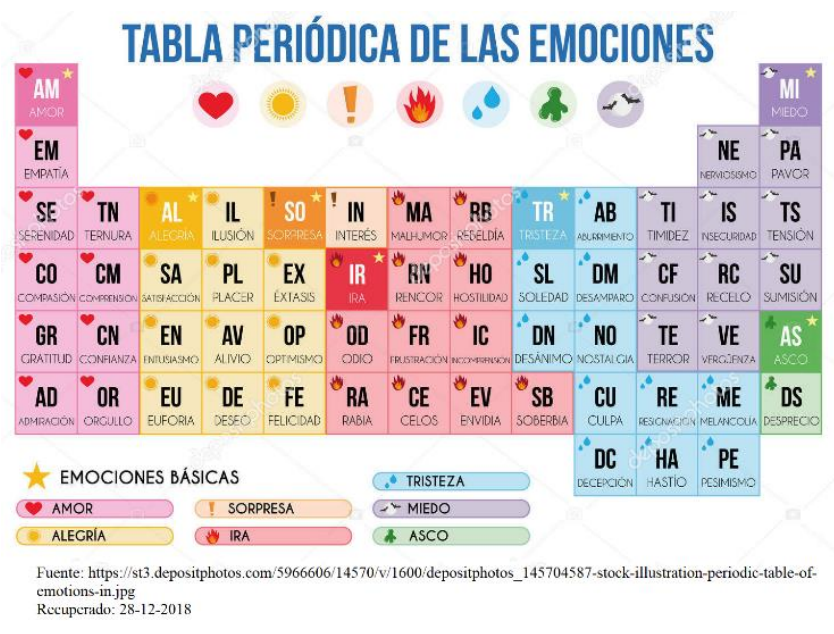


El hombre es el HOMO SAPIENS, el hombre que sabe, el hombre sabio. (...) el hombre por naturaleza (y por cronología evolutiva) primero siente, luego piensa. Es un "hombre sentidor", emocional antes que sabio. (...) Primero se siente (miedo, alegría, cólera, dolor) y luego irán apareciendo los procesos cognitivos superiores como la imaginación, el análisis, la deducción y la razón (Arboccó de los Heros, 2015).

El aprendizaje es un proceso que se relaciona con los cambios a nivel neuronal, cognitivo, emocional y conductual como resultado de la experiencia, lo que permite que la persona se adapte fácilmente a su entorno (Campos, 2010). "El proceso de aprendizaje modela el cerebro" (Di Gesù y Seminara, 2012, p.7), y el proceso de enseñanza aprendizaje a decir de De la Cuesta $(2016$, p.10) es un nexo interdisciplinario en el que "se puede ver cómo el aprendizaje, un proceso cognitivo y abstracto, y el cerebro, el órgano más complejo del ser humano, son co-creadores, uno del otro, en una interacción mutua".

Los cerebros humanos son distintos ya que el aprendizaje cambia su estructura, cuanto más aprendemos más complejo se vuelve nuestro cerebro. Los mejores aprendizajes incluyen curiosidad, anticipación y desafío (Velásquez et al., 2009).

Las investigaciones sobre el cerebro y específicamente sobre las emociones refuerzan la idea de que la enseñanza debe tomar en cuenta tanto las emociones como los sentimientos ya que al hacerlo se intensifican la actividad de las redes neuronales y por tanto las conexiones sinápticas. La Neurobiología muestra evidencias de que se aprende mejor cuando se involucran emociones y sentimientos en este hecho.

"Las emociones son cruciales en los procesos de aprendizaje. Lo que aprendemos está influenciado y organizado por emociones y estructuras mentales basadas en expectativas, inclinaciones y prejuicios personales, grados de autoestima y la necesidad de interactuar socialmente" (Salazar, 2005, p. 13).

La escuela es el sitio ideal para el aprendizaje ya que permite la socialización entre personas de la misma edad, lo que provoca cambios cerebrales que se transforman en conductas distintivas (Mora, 2013).

El proceso de aprendizaje involucra todo el cuerpo y el cerebro, actúa como una estación receptora de estímulos y se encarga de seleccionar, priorizar, procesar información, registrar, evocar, emitir respuestas motoras, consolidar capacidades, entre otros miles de funciones. El cerebro es el único 
órgano del cuerpo humano que tiene la capacidad de aprender y a la vez enseñarse a sí mismo (Falco y Kuz, 2011, p. 3).

Las diversas estimulaciones cerebrales pueden producir diferentes resultados y, por ello, se plantean distinciones entre los aprendices; por ejemplo, los kinestésicos necesitan mayor movimiento; los auditivos requieren conversar sobre el material (Sprenger, citado por Salazar, 2005).

La enseñanza es una tarea multifacética que permite a los estudiantes expresarse en forma diferente: visual, táctil, emocional, entre otros. Aquí no es distinguir "tipos de inteligencia", sino más bien, el reconocimiento de que los estilos de aprendizaje tienen su base también en la configuración neuronal de cada persona. (Salazar, 2005, p.17).

El aprendizaje se considera como un aspecto de la plasticidad neuronal, puesto que es la modificación de la conducta de la persona, a partir de experiencias previas; ello indica que el cerebro aprende y debido a su plasticidad se modifica produciéndose cambios sinápticos de orden anatómico y funcional (Velásquez, et al., 2009, p. 332).

La neuroplasticidad es "la capacidad que tiene el cerebro de cambiar su estructura y los patrones de actividad (...). Ese cambio puede producirse como consecuencia de las experiencias y de los propios pensamientos" (Davison y Begley, 2012, p.149).

El aprendizaje es modificado por las emociones, ya que éstas afectan a la conducta y desencadenan los cambios químicos que regulan los cambios de ánimo; es “constructivo, ya que los procesos utilizados permiten la construcción de significados a partir de establecer relaciones entre la información almacenada en la memoria y la nueva información" (Pozo, 1998; citado en Abate, 2006, p.6); es una función fundamental de las neuronas que no se puede llevar a cabo de modo individual, sino que requiere grupos de neuronas" (Greenfield , 1995; citado en Arias, 2011, p.13). Así el aprendizaje es el resultado de la comunicación interneural, mientras mayor es la comunicación, mayor es el aprendizaje (Velásquez, et al., 2009). Cuando un nuevo estímulo llega al cerebro se desencadena el proceso de aprendizaje, el estímulo puede ser interno o externo, una lluvia de ideas o una nueva experiencia; el estímulo se distribuye y procesa a varios niveles con lo que se crea una memoria potencial (Arias, 2011).

El aprendizaje favorece la formación de redes conceptuales de conocimiento que se almacenan en la memoria de largo plazo, y dependen de qué tan significativo es el nuevo aprendizaje para mantenerse. Para Wolf (2001) los mecanismos de almacenamiento se pueden describir como redes de asociaciones, así los nuevos 
aprendizajes para mantenerse deben configurarse como redes de asociaciones significativas, aprendizajes nuevos relevantes para el aprendiz, ligados a conocimientos previos almacenados en su memoria de largo plazo.

El aprendizaje se produce cuando una célula requiere menos impulsos de otra, la siguiente vez que se activa, ya que los aprendizajes nuevos necesitan de varias estimulaciones hasta que cambian su conducta (Donald Hebb, citado en Arias, 2011). El aprendizaje es mayor cuando el ambiente en el que se desarrolla es fluido y variado, ya que las redes neuronales son excitadas en ambientes desconocidos y cambiantes (Velásquez et al., 2009).

El aprendizaje se bloquea por las emociones negativas como ansiedad, furia o depresión entre otras, ya que estas emociones desvían la atención y entorpecen la concentración, lo cual deriva en la paralización de la memoria activa, es decir que se impide la retención en la mente de la información relacionada con la tarea que se está realizando (Casas 2003: citado por Velásquez et al., 2009). El aprendizaje se inhibe además por la falta de atención lo cual incide en el desarrollo total del cerebro (Jensen, 2004; citado por Velásquez et al., 2009).

Los principios de aprendizaje del cerebro según Caine y Caine (1997, citado en Salas, 2003) son:

1. El cerebro es un complejo sistema adaptativo, es plástico y mejora con el aprendizaje.

2. El cerebro es un cerebro social, requiere de un entorno enriquecido socialmente para mejorar.

3. La búsqueda de significación es innata, la curiosidad es un importante estimulador para aprender.

4. La búsqueda de significado ocurre a través de pautas, el aprendizaje se da de mejor manera cuando se utilizan esquemas o mapas mentales.

5. Las emociones son críticas para la elaboración de pautas: lo que aprendemos es influido por las emociones.

6. Cada cerebro simultáneamente percibe y crea partes y todos, tiene un enfoque holístico.

7. El aprendizaje implica tanto una atención focalizada como una percepción periférica, el aprendizaje es implícito y explícito.

8. El aprendizaje siempre implica procesos conscientes e inconscientes. 
9. Tenemos al menos dos maneras de organizar la memoria: sistemas para recordar información no relacionada (taxonómicos) motivada por premio y castigo. El otro sistema es el espacial/autobiográfico que permite el recuerdo de experiencias, motivada por la novedad.

10. Cada cerebro está organizado de manera única.

11. El aprendizaje es un proceso de desarrollo mediado por la experiencia.

12. El aprendizaje complejo se incrementa por el desafío y se inhibe por la amenaza.

Estos principios son importantes para lograr aprendizajes duraderos basados en las emociones de los estudiantes, léase intereses, motivaciones personales, conocimientos previos, experiencias de vida, objetivos personales y otras que permiten la repetición de estímulos que provocan el reforzamiento de las redes neuronales y su consiguiente automatización, es decir aprendizaje.

\subsection{La didáctica y neurodidáctica}

\section{LA NEURODIDÁCTICA}

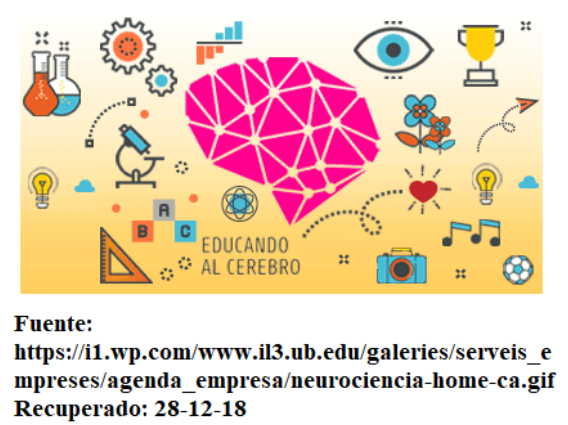

"La Didáctica es el método y sus tareas son la enseñanza y el aprendizaje" (Di Gesú y Seminara, 2012, p.16); "el aprendizaje puede ser visto como el resultado de la creación de una memoria potencial impulsada por el estímulo, que la interacción con el entorno procura potenciar o inhibir (Di Gesú, 2012). "La neurodidáctica está localizada en el espacio transfronterizo de las ciencias educativas y la neurología" (De la Cuesta, 2016, p.10); organiza e implementa la interacción entre docente y aprendiz; "el objetivo de la Neurodidáctica es la creación de metodologías didácticas para una ecología de la mente" (Di Gesú y Seminara, 2012).

La neurodidáctica como concepto fue definida en 1988 por primera vez por Gerhard Friedrich y Gerhard Preiss en una revista alemana de neurología, quienes propusieron como objetivo fundamental de esta nueva ciencia, que los estudiantes 
aprendan en función de sus dotes y talentos (Gerhard y Gerhard, 2004, p. 45). La neurodidáctica "Supone un nuevo campo de investigación que persigue encontrar la manera más eficaz de enseñar mediante la utilización de las contribuciones neurocientíficas más significativas aplicadas a la educación” (Fernández, 2017).

A las nuevas formas de enseñar basadas en el conocimiento del cerebro Paniagua (2013) denomina neurodidáctica, y afirma que su objetivo es diseñar estrategias didácticas y metodológicas que promuevan un mayor desarrollo cerebral y por tanto un mayor aprendizaje; las estrategias que el docente diseñe pueden incidir positiva o negativamente en el aprendizaje según estas lo inhiban o motiven, es decir que el docente tiene la posibilidad de modificar el cerebro de sus estudiantes si utiliza las estrategias didácticas apropiadas, así una estrategia que despierte interés, como un debate o la resolución de problemas no excesivamente complicados puede promover una actividad eléctrica de entre 12.5 y 25 ciclos por segundo, mientras que las estrategias basadas en la repetición pueden bajar la actividad eléctrica a 7 ciclos por segundo, provocando sueño en los estudiantes (Panigua, 2013).

De lo que se deduce que hay que emocionar para enseñar, y el aprendizaje tiene que ver con la supervivencia, de allí que la curiosidad, emoción básica, es requerida para iniciar un proceso de aprendizaje y por tanto debe ser tomada en cuenta por los educadores para plantear estrategias didácticas que posibiliten aprendizajes duraderos (Mora, 2004; citado por Tristan, 2012).

"La Neurodidáctica, viene a ser una conjunción entre pedagogía, epistemología, psicología, neurología, ciencias cognitivas, y aprendizaje: Ciencias y disciplinas interactuando de la mejor manera en la búsqueda de aprender, facilitar y organizar la enseñanza misma” (Gómez y Escobar, 2015, p.5); según Meléndez (2009), se ocupa además del estudio de las funciones ejecutivas en tanto son fundamentales para el proceso de aprendizaje, ya que son:

El conjunto de habilidades cognitivas que permiten la anticipación y el establecimiento de metas, el diseño de planes y programas, el inicio de las actividades y de las operaciones mentales, la autorregulación y monitoreo de las tareas, la flexibilidad en el trabajo cognitivo y su organización en el tiempo y el espacio" (Ustarróz y MuñozCéspedes 2005, citado en Meléndez, 2009, p. 9). 


\subsection{Las estrategias didácticas y el trabajo docente}

"En el campo de la pedagogía, las estrategias didácticas se refieren a tareas y actividades que pone en marcha el docente de forma sistemática para lograr determinados objetivos de aprendizaje en los estudiantes" (Rodríguez, 2007, p.3).

"El concepto de estrategias didácticas se involucra con la selección de actividades y practicas pedagógicas en diferentes momentos formativos, métodos y recursos en los procesos de Enseñanza - Aprendizaje" (Velasco y Mosquera, 2010, p.1).

Los nuevos conocimientos producen nuevas conexiones sinápticas, por lo que las estrategias que se utilicen deben fomentar la curiosidad, deben ser excitantes, novedosas y significativas para los estudiantes, a fin de que el cerebro las vincule con redes conceptuales ya almacenadas en la memoria de largo plazo.

El uso en el aula de estrategias didácticas que promuevan las clasificaciones, análisis, pruebas y profundizaciones permiten brindar retroalimentación sobre lo bien que se están dando las asociaciones entre redes conceptuales (Salazar, 2005).

Entre otras estrategias didácticas importantes están: la realización de ejercicios de memorización de imágenes, listas de nombres, listas de series numéricas, etc.

El cerebro enriquece su aprendizaje, cuando se le presentan retos en un entorno desafiante, esta estrategia permite la generación de nuevas conexiones dendríticas (Jensen, 2010).

En el año 2013 panelistas en la Universidad de Stanford explicaban que la utilización de juegos como una herramienta educacional provee oportunidades para un aprendizaje más profundo, porque resulta que los juegos son difíciles y lo que se busca desde las neurociencias es potenciar la generación del entramado de conexiones neuronales, posibilitando que el alumno genere nuevos caminos para resolver problemas (...) el juego dirigido (en inglés, playful learning) es un componente fundamental del aprendizaje, debido a que ayuda a "enfocar" el interés de los alumnos (Falco y Kuz, 2011).

El juego es importante como estrategia de aprendizaje porque permite el autoconocimiento y fomenta la curiosidad con lo cual se abre una puerta a la emoción y por tanto al aprendizaje. El juego es una herramienta para conocerse y conocer el entorno, con lo que se potencia las relaciones inter e intra personales, fundamentales para activar el sistema cognitivo. 
Otra forma de potenciar el aprendizaje es a partir del estímulo al proceso de lectoescritura, mismo que debe convertirse en una práctica cotidiana que permite al cerebro realizar nuevas conexiones sinápticas que refuercen las habilidades o conocimientos adquiridos.

Una estrategia importante para preparar al cerebro para el aprendizaje es la estimulación motora, ya que cuando el cuerpo trabaja mejor, el cerebro se prepara para dar respuestas rápidas a las necesidades que se presenten, así el ejercicio constante permite la generación de una substancia que fomenta la capacidad de que las neuronas se conecten entre sí.

Los neurocientíficos italianos Giacomo Rizzolatti, Leonardo Fogassi y Vittorio Gallese (Arboccó de los Heros, 2015), de casualidad descubrieron las denominadas neuronas espejo, que se activan cuando una persona observa o se imagina una acción realizada por otra. Según Arboccó de los Heros (2015b), “este descubrimiento (...) permite tener una mejor explicación evolutiva y cerebral para una serie de aspectos como, el aprendizaje, la imitación, la identificación y la empatía —entre otros — tan importantes para la vida y sobre todo para la vida social”.

La inteligencia emocional determina la manera en que nos relacionamos y entendemos el mundo; tiene en cuenta actitudes, sentimientos y engloba habilidades como el control de los impulsos, la autoconciencia, automotivación, confianza, entusiasmo y empatía (Carvajal, 2013).

La Inteligencia emocional propuesta por Goleman (1996), da cuenta de la necesidad de enseñar a los estudiantes sobre el autoconocimiento y el auto control, sobre el manejo de sus emociones y la necesidad de pensar sobre nuestro pensamiento, cómo pensamos y sobre nuestra memoria, cómo memorizamos, es decir sobre la estrategia de metacognición y metamemoria.

Eliminar la amenaza y el estrés causados por la humillación o vergüenza por parte de profesores o compañeros es muy importante, para ello los docentes deben enseñar a los estudiantes a planificar el tiempo, las técnicas de respiración, las habilidades de relación interpersonal, el apoyo de los pares, los juegos, ejercicios grupales, debates, celebraciones, así como crear un ambiente de aula favorable que evite la intimidación y permita establecer un ambiente democrático en donde los estudiantes puedan manifestarse libremente (Salas, 2003; citado por Velásquez et al., 2009). 
Los estudiantes deben aprender a educar sus emociones, es decir a identificarlas, reflexionar sobre ellas, encontrar su origen, guiarlas y finalmente a automotivarse.

\title{
1.9. El método tradicional de enseñanza y el desarrollo del cerebro
}

\author{
"La escuela tradicional no favorece ni la comprensión, ni la formación valorativa de los \\ individuos. El autoritarismo inhibe, acompleja, genera inseguridad y dogmatiza al individuo. El \\ mecanicismo por su parte orienta la actividad del niño hacia los aprendizajes arbitrarios y no \\ significativos" (De Zubiría, 1999).
}

Los métodos de enseñanza tradicionales propician en los estudiantes la pasividad, poca reflexión, y fomentan la memorización; no propician la investigación elemental, ni la investigación científica; los aprendizajes que bajo este método se producen son poco significativos y se olvidan fácilmente. El objetivo del método tradicional es la transmisión de conocimientos, por lo que no hace énfasis en la construcción individual ni colectiva del aprendizaje por parte de los estudiantes. Por otro lado, los métodos tradicionales no toman en cuenta los intereses de los estudiantes, es decir no les preocupa el tipo de emociones que pueden despertar en los aprendices, que podrían ser de rechazo o indiferencia.

"El alumno es tratado como el lugar de una acción que se ejerce en él desde el exterior, por tanto, el alumno tiene estatuto de objeto" (Not, 1979). La escuela tradicional no diferencia a los estudiantes según sus necesidades, talentos o intereses, parte del supuesto que todos son iguales y por tanto aprenden igual. Así se considera que todos los estudiantes tienen el mismo nivel de conocimientos, no se toma en cuenta los conocimientos previos de cada uno, ni sus posibilidades individuales y diferenciadas de superación.

El desarrollo de habilidades cognitivas requiere de la intervención positiva de las emociones, la atención y motivación. En este contexto es importante destacar la función motivadora de los recursos didácticos, "si bien puede ser temporal hasta que los alumnos se habitúen a ellos, el color, el movimiento, el sonido, la diagramación son otros tantos elementos que llaman la atención y la focalizan hacia los contenidos objeto de aprendizaje" (Colom, et.al, 2002); 
La escuela tradicional tiene como objetivo principal la transmisión de conocimientos de quien sabe, a quien ignora, maestro-alumno, no el desarrollo de habilidades cognitivas en los estudiantes, a excepción de la memoria, por lo que sus métodos no favorecen de manera explícita el desarrollo del cerebro de los estudiantes.

\section{Conclusiones}

Las emociones como se ha visto a lo largo de la presente revisión bibliográfica son básicas para lograr aprendizajes duraderos, por lo que son fundamentales para la neurodidáctica, ya que esta ciencia en ciernes debe desarrollar estrategias de aprendizaje que tomen en cuenta el manejo y desarrollo de las emociones por parte de docentes y estudiantes.

El conocimiento de cómo se producen las emociones, y cómo se pueden manifestar a partir de diferentes sentimientos, es fundamental por parte de docentes y estudiantes, a fin de que aprendan que es posible administrarlas, lo que requiere de un claro manejo de la inteligencia emocional, específicamente del auto conocimiento y autocontrol o auto regulación relacionados ambos con el lóbulo frontal, encargado del juicio, control de impulsos y planificación.

Para que el aprendizaje sea efectivo es necesario que el ambiente emocional del aula sea lo más positivo posible, que el cerebro emocional de los estudiantes y la amígdala en específico, estén listos para captar los estímulos provenientes del entorno, mismos que deben ser positivos para que los nuevos conocimientos se adquieran fácilmente, por lo que los docentes deben manejar estrategias didácticas que se basen en el conocimiento de cómo aprende el cerebro, es decir que se basen en los principios de la neuroeducación y neurodidácticas.

Las emociones pueden bloquear el intento de fijar nuevos conocimientos si éstos no son interesantes, o si avivan recuerdos perturbadores, a la vez pueden potenciar los aprendizajes si éstos provocan sensaciones gratificantes, ya que las personas actúan en consecuencia de sus emociones, ellas intensifican los deseos de persistir en actos inclusive heroicos o de abandonar cualquier acción al sentirse desolados; lo que implica el manejo de estrategias didácticas propuestas por la neuroeducación; Manejar este conocimiento es importante para la docencia, ya que permite reflexionar sobre los mejores métodos que 
deben ser utilizados a fin de que a los estudiantes les resulte agradable el proceso de aprendizaje.

Las estrategias didácticas más efectivas están relacionadas con la neuroeducación, y se basan en actividades que permiten a partir de la reflexión sobre cómo se aprende, proponer ejercicios que toman en cuenta los intereses de los educandos, y despiertan su atención y motivación intrínseca, las mismas que propician aprendizajes más duraderos. Estas estrategias pueden ser actividades lúdicas, físicas, trabajos que impliquen la interrelación social y el autoconocimiento, para lograr un buen manejo de las emociones permitiendo así el desarrollo tanto de la inteligencia cognitiva como emocional.

Lo expuesto conlleva a la necesidad de que los docentes deban conocer las bases y principios neurobiológicos del cerebro y su desarrollo cognitivo, a fin de que su práctica educativa esté sustentada en bases científicas.

Cuando los estudiantes no están motivados, el aprender les resulta indiferente y esta conducta puede convertirse en permanente. Lo dicho implica una responsabilidad de los educadores a la hora de enseñar, sus propuestas pedagógicas deben estar integradas por estrategias que tomen en cuenta las necesidades emocionales de los aprendices.

La escuela tradicional no favorece mayoritariamente el desarrollo de las habilidades cognitivas de los estudiantes, trabaja principalmente en el desarrollo de la memoria, aunque ese no sea su objetivo. Las habilidades de análisis, síntesis y habilidades emocionales como la interacción social son dejadas de lado, sin embargo de su importancia para la formación integral de los estudiantes.

El estudio realizado requiere una mayor profundización en el análisis de las didácticas especiales a utilizar según áreas de estudio y edades de los aprendices, ya que tanto su motivación como su foco de atención puede variar de acuerdo a estos factores.

Del presente estudio se desprenden algunos temas de investigación importantes para la enseñanza y el aprendizaje, tales como el desarrollo de estrategias para la enseñanza a personas que viven en ambientes emocionales frágiles, que actualmente representan porcentajes elevados, dados los crecientes problemas socioeconómicos que atraviesan nuestros países. 


\section{Referencias}

Abate, N. 2006. La Psicología Cognitiva y sus aportes al proceso de aprendizaje.

Recuperado de:

http://www.quadernsdigitals.net/datos_web/hemeroteca/r_1/nr_804/a_10845/108 $\underline{45 . h t l m}$

Aguilar, J. (2011). La estructura del sistema nervioso. México: Asociación Oaxaqueña de Psicología A.C. Recuperado de: http://cleuadistancia.cleu.edu.mx/cleu/flash/PAG/lecturas/poligrafia/Estructura\% 20sistema\%20nervioso.pdf

Arboccó de los Heros, M. (2015). Apuntes psicoeducativos a la luz de las neurociencias. Universidad Femenina del Sagrado Corazón, 11(1), 7-15. Lima, Perú.

Recuperado de:

http://www.unife.edu.pe/publicaciones/revistas/revista_tematica_psicologia_201 5/7ARBOCCO.pdf

Arias, D. (2011). Módulo: Cerebro y aprendizaje. Fundación Universitaria del Área Andina, Centro de Educación a Distancia. Recuperado de: https://expedicionatlantida.files.wordpress.com/2011/04/cerebro-aprendizaje-2lilianaarias2.pdf

Bajo, T., Fernández, A., Ruiz, M., Gómez-Ariza, C. (2016). Memoria, estructura y funciones, 167-197. Recuperado de: https://www.researchgate.net/publication/295858472_Memoria_estructura_y_fu nciones

Blakemore, S-J., Frith, U. (2010). Cómo aprende el cerebro, las claves para la educación. Barcelona: Editorial Planeta.

Bruer, T. (2016). Neuroeducación un panorama desde el puente. Propuesta educativa. Revista FLACSO Argentina. Recuperado de: http://www.scielo.cl/result.html?cx=002778039995109192455\%3Amjmfr2dvt3c 
\&cof=FORID\%3A11\&q=neuro+educacion\&sa=Search\&siteurl=www.scielo.cl \%2F\&ref=www.google.com.ec\%2F\&ss=5365j4591559j15

Campos, A. (2010). Neuroeducación: uniendo las neurociencias y la educación en búsqueda del desarrollo humano. La educación, Revista Digital, 143, 1-14. Recuperado de: http://www.educoea.org/portal/La_Educacion_Digital/laeducacion_143/articles/n euroeducacion.pdf

Campos, A. (2014). Los aportes de la neurociencia a la atención y educación de la primera infancia. Centro Iberoamericano de Neurociencia, Educación y Desarrollo Humano. Cerebrum Ediciones. Recuperado de: https://www.unicef.org/bolivia/056_NeurocienciaFINAL_LR.pdf

Carvajal, C. (2013). Creatividad e intuición en la praxis metodológica, reflexión a la luz de la neurociencia cognitiva. Revista TELOS. 15 (1), 77-90. Recuperado de: http://publicaciones.urbe.edu/index.php/telos/article/viewArticle/2503/3652

Codina, M. (2014). Neuroeducación en virtudes cordiales. Recuperado de: http://www.academia.edu/12975357/Tesis_doctoral_Neuroeducaci\%C3\%B3n_e n_virtudes_cordiales._Una_propuesta_a_partir_de_la_Neuroeducaci\%CC3\%B3n_ y_de_la_\%C3\%A9tica_discursiva_cordial

Codina, M. (2015). Neuroeducación en virtudes cordiales: cómo reconciliar lo que decimos con lo que hacemos. Barcelona: Octaedro. Recuperado de: https://www.octaedro.com/appl/botiga/client/img/16075.pdf

Colom, A., Bernabeu, J., Domínguez, E.,Sarramona, J. (2002). Teorías e instituciones contemporáneas de la educación. Barcelona: Ariel Educación.

Davison, R., Begley, S. (2012). Claves para modificar nuestras reacciones y mejorar nuestras vidas. Grupo Planeta.

De la Cuesta, I. (2016). Neurodidáctica aplicada. Recuperado de: www.divaportal.org/smash/record.jsf?pid=diva2:944465 
De la Barrera, M L., Donolo, D. (2009). Neurociencias y su importancia en contextos de aprendizaje. Revista digital Universitaria, 10 (4), 1-17. Recuperado de: http://www.revista.unam.mx/vol.10/num4/art20/art20.pdf

De Zubiría, J. (1999). Las vanguardias pedagógicas. Loja: Editorial de la Universidad Técnica Particular de Loja.

Di Gesu, F., Seminara. A. (2012). Neurodidáctica y la implicación de emociones en el aprendizaje. LynX. Panorámica de Estudios Lingüísticos, 11, 5-39. Recuperado de:

https://www.researchgate.net/profile/Floriana_Di_Gesu/publication/260187668_

Neurodidactica_y_la_implicacion_de_emociones_en_el_aprendizaje/links/02e7e $\underline{\text { 52ffaf01bb698000000/Neurodidactica-y-la-implicacion-de-emociones-en-el- }}$ aprendizaje.pdf

Di Gesú, F. (2012). Neurodidáctica y aprendizaje de lenguas afines: español e italiano.LynX. Recuperado de: https://www.researchgate.net/profile/Floriana_Di_Gesu/publication/260187741_ Neurodidactica_y_aprendizaje_de_lenguas_afines_espanol_e_italiano/links/0c96 052ffac504ddf9000000/Neurodidactica-y-aprendizaje-de-lenguas-afinesespanol-e-italiano.pdf

Falco, M., Kuz, A. (2016). Comprendiendo el Aprendizaje a través de las Neurociencias, con el entrelazado de las TICs en Educación. Buenos Aires. Recuperado de: https://es.scribd.com/document/356120355/Comprendiendo-ElAprendizaje-a-Traves-de-Las-Neurociencias-Con-El-Entrelazado-de-Las-TICsen-Educacion

Fernández, A. (2017). Neurodidáctica e inclusión educativa. Revista Profesional de Docencia y Recursos Didácticos, 91, 262-266. Recuperado de: http://publicacionesdidacticas.com/hemeroteca/articulo/080051

Freilich, M. (1989). El cerebro triuno. Diario El Nacional. Recuperado de: https://es.scribd.com/document/120916672/cerebro-triuno 
Gerhard, F., Gerhard, P. (2003). Neurodidáctica, Revista Mente y cerebro: 4 Esquizofrenia, España. Recuperado de: https://www.investigacionyciencia.es/revistas/mente-y-cerebro/esquizofrenia$\underline{356 / \text { neurodidctica-3910 }}$

Gluck, M., Mercado, E. (2009). Aprendizaje y memoria. Mexico: Mc. Graw Hill.

Goleman, D. (1996). La Inteligencia Emocional. México: Ediciones S. A.

Gómez, J., Escobar, M. (s.f.). Neurodidáctica y educación. Una aproximación desde las humanidades incluyendo la literatura. Recuperado de: http://soda.ustadistancia.edu.co/enlinea/congreso/congresoedu/2\%20Pedagogia\% 20y\%20dida\%B4ctica/2\%2017\%20Neurodidactica\%20y\%20educacion.pdf

Jensen, E. (2010). Cerebro y aprendizaje. Competencias e implicaciones educativas. Madrid: Ediciones Narcea S. A. Recuperado de: http://memsupn.weebly.com/uploads/6/0/0/7/60077005/cerebro_y_aprendizaje e. jensen.pdf

LeDoux, J. (1999). El cerebro emocional. Barcelona: Editorial Planeta.

Labus, C., Romero, E. (s.f.) Neurociencia, memoria, aprendizaje y educación. Recuperado de: http://www.dfpd.edu.uy/cerp/cerp_norte/informacion/201309matjorn/NEUROCI ENCIA\%20(1).pdf

Maureira, F. (2010). Neurociencia y educación. Recuperado de: https://www.researchgate.net/profile/Fernando_Maureira_Cid/publication/27132 $\underline{8225 \_N e u r o c i e n c i a \_y \_e d u c a c i o n / l i n k s / 54 c 57 b f b 0 c f 219 b b e 4 f 50411 / N e u r o c i e n c i}$ a-y-educacion.pdf

Meléndez, L. 2009. Neurodidáctica y el desarrollo de las funciones ejecutivas. VIII Congreso Educativo: El sentido de la Educación en un Mundo en Crisis. Universidad Interamericana de Costa Rica. Recuperado de: https://www.google.com.ec/search?ei=YHOMWv71Osb0zgKUyKWoCA\&q=m

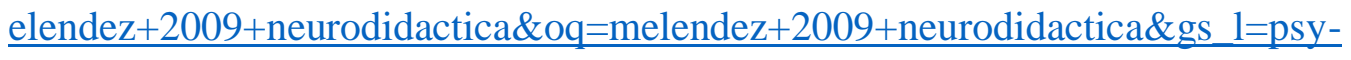


ab.3..33i160k1.588779.594984.0.596079.25.25.0.0.0.0.232.3048.0j20j1.21.0....0. ..1c.1.64.psy-ab..4.19.2738...35i39k1.0.UCciAm1xgSY

Mogollón, E. (2010). Aportes de las neurociencias para el desarrollo de estrategias de enseñanza y aprendizaje de las Matemáticas. Revista Electrónica Educare, XIV(2), 113-124. Recuperado de: file:///C:/Users/Veronica/Downloads/DialnetAportesDeLasNeurocienciasParaElDesarrolloDeEstrate-3642017.pdf

Mora, F. (2013). La neuroeducación demuestra que emoción y conocimiento van juntos. El País, Blogs Sociedad. Recuperado de: http://blogs.elpais.com/ayuda-alestudiante/2013/12/la-neuroeducacion-demuestra-que-emocion-y-conocimientovan-juntos.html

Morgado, I. (2005). Psicobiología del aprendizaje y la memoria. Cuadernos de Información y Comunicación, 10, 221-233. Recuperado de: file://C:/Users/Veronica/Downloads/8143-8226-1-PB\%20(1).PDF

Paniagua, M. (2013). Neurodidactica: una nueva forma de hacer educación. Recuperado de: http://www.scielo.org.bo/scielo.php?pid=S2071$\underline{081 X 2013000100009 \& \text { script }=\text { sci_abstract }}$

Rodríguez, R. (2007). Compendio de estrategias bajo el enfoque de competencias. Recuperado de: http://www.itesca.edu.mx/documentos/desarrollo_academico/compendio_de_estr ategias_didacticas.pdf

Salas, R. (2013). ¿La educación necesita realmente de la Neurociencia?. Estudios pedagógicos, 29, 155-171. Recuperado de: https://scielo.conicyt.cl/scielo.php?script=sci_arttext\&pid=S0718$\underline{07052003000100011}$

Salazar, S. (2005). El aporte de la neurociencia para la formación docente. Actualidades Investigativas en Educación, 5(1), 1-19. Universidad de Costa Rica. Recuperado de: https://revistas.ucr.ac.cr/index.php/aie/article/view/9116 
Souza, D. (2017). El Cerebro reconectado. España. Recuperado de: https://issuu.com/sergiocristerna/docs/neurociencia_educativa_mente_cereb

Totger, M. (2017). Neurociencias y Neuroaprendizajes: las emociones y el aprendizaje, nivelar estados emocionales y crear un aula con cerebro. Córdoba: Editorial Brujas.

Tristan, M. (2012). La neuroeducación será el eje de la docencia del futuro.

Recuperado de:

https://rosamtristan.files.wordpress.com/2012/11/escuelafranciscomora.pdf

Velasco, M., Mosquera, F. (s. f.). Estrategias didácticas para el aprendizaje colaborativo. Recuperado de:

http://acreditacion.udistrital.edu.co/flexibilidad/estrategias_didacticas_aprendizaj e_colaborativo.pdf

Velásquez, B., Remolina N., Calle, M. (2009). El cerebro que aprende. Tabula Rasa, 11: 329-347. Recuperado de: http://www.scielo.org.co/pdf/tara/n11/n11a14.pdf

Wolfe, P. (2007). Brain Research and Education: Fad or Foundation? Recuperado de: http://commons.emich.edu/cgi/viewcontent.cgi?article=1001\&context=loexconf2 $\underline{007}$ 